\title{
Exercise-Induced Acute Compartment Syndrome in Bilateral Upper Extremities: A Clinical Case
}

\author{
Raymond E. Kennedy, $\mathrm{MD}^{1^{*}}$, Mark K. Lane, $\mathrm{MD}^{2}$ \\ ${ }^{1}$ Department of Surgery, Hackensack Meridian Health, Palisades Medical Center, Hackensack, NJ, USA \\ ${ }^{2}$ Department of Orthopaedics and Rehabilitation, Detroit Medical Center, Wayne State University School of \\ Medicine, Orthopaedic Surgery Residency Program, Detroit, MI, USA \\ *rkennedy@neomed.edu
}

Abstract: Compartment syndrome is developed by a build-up of pressure in an enclosed space in the body, and can be caused by trauma, medical conditions that alter fluid balance, exercise, or can be iatrogenic. A decrease in the microcirculation pressure gradient causes local ischemia, making compartment syndrome a true medical and surgical emergency often requiring a fasciotomy to reduce intra-compartmental pressures and restore microscopic flow. A 22-year-old female presented with bilateral upper extremity pain and swelling after vigorous exercise and her exam revealed a decreased range of motion, tense skin over the affected areas, as well as rising CPK levels, which led to the early diagnosis of acute compartment syndrome. Surgical release was immediately performed bilaterally with delayed primary closure. The patient regained full function and range of motion in both arms, without any short or long-term complications. Acute compartment syndrome is a limb-threatening emergency. Rapid diagnosis and early surgical treatment with fasciotomy is necessary to regain function and circulation to the affected area, and effectively save the limb. Delayed diagnosis and/or surgical treatment puts the patient and the affected limb at risk, and even with eventual treatment, allows for the development of complications such as Volkmann's contractures and acute renal failure.

\section{INTRODUCTION}

Acute compartment syndrome (ACS) is generated by rapidly increasing intracompartmental pressures, often associated with trauma [1]. In contrast, chronic compartment syndrome is said to occur from excessive exercise or strain in the extremities [1]. When the tissue pressure comes within $25-30 \mathrm{mmHg}$ of diastolic blood pressure microperfusion ceases, and muscle and nerve ischemia are the result [2]. Pain and decreased sensation secondary to nerve irritation arise from the accumulation of waste products from cell metabolism and breakdown, as well as the lack of oxygenated blood. This causes further swelling, and at some point becomes a self-aggravating cycle perpetuating the compartment syndrome.

Exercise-induced compartment syndrome of the hand and upper extremity is a rare and uncommon condition with limited literature and research [3]. Compartment syndrome is well described for the lower extremities, but only a few reports on upper limb exercise-induced compartment syndrome exist in literature, as is described in our case [4]. We present a case of a 22-year-old female with exercise-induced, bilateral upper extremity acute compartment syndrome requiring emergent bilateral fasciotomies with delayed primary closure.

\section{CASE}

A 22-year-old female came to the emergency department complaining of right arm swelling for the past two days. She had been working out "exceptionally hard", without having prior athletic training. Although her arms were sore from the exercise, she then went to work for the day. Continually increasing pain during the day prompted her to visit the ER. She stated that her arms became tender, causing difficulty in flexing and extending 


\section{Exercise-Induced Acute Compartment Syndrome in Bilateral Upper Extremities: A Clinical Case}

her elbows due to the pain and swelling in her upper arms. She rated the pain a 6/10, described it as sharp, and being exacerbated by movement. Her left arm was beginning to swell as well, and ibuprofen had not relieved any of the symptoms. She denied any chest pain, dyspnea, history of clotting disorders or other symptoms. She also stated that she had a tingling sensation when she tried to carry anything with her arms.

On physical examination, there was limited range of motion with passive and active flexion/extension in the right arm due to pain. Her right arm measured $34 \mathrm{~cm}$, and her left arm measured $32 \mathrm{~cm}$. Both upper arms were tense/ hard to palpation with some altered subjective sensation on the right. Radial pulses were normal bilaterally with a normal neuromotor exam throughout except for very weak and painful elbow flexion/extension, and forearm supination.

Vitals: Blood Pressure: 132/80 Pulse: 73, Respiratory rate: 16, Temperature: 98.3, Sa02: 98\% on room air. A complete blood count showed a white blood cell count of 11.6. CPK levels were elevated at 15,604, and a urinalysis showed trace ketones, large blood, 5-10 red blood cells, 1+ bacteria and moderate squamous epithelial cells. These results are consistent with myoglobinuria.

A bilateral upper extremity ultrasound was negative for deep venous thrombosis. She was given a $1 \mathrm{~L}$ bolus of normal IV saline and 30mg IV of Toradol for pain. Orthopaedic surgery was consulted and a bicarbonate drip was initiated.

Upon orthopaedic evaluation in the emergency room, the patient was taken urgently to the operating room for fasciotomies of the anterior and posterior compartments of both upper arms only, and left "open" for delayed closure. A laced vessel loop/staple construct was utilized to prevent unnecessary skin retraction and facilitate delayed primary closure. Intraoperative findings showed the musculature of the anterior compartments of the upper arms to be dusky and violaceous in appearance upon opening of the compartment, but still all contractile with stimulation with the electrocautery. By the time the posterior compartments were decompressed, the color of the anterior compartments were already improving and the compartments were softening. The forearms remained soft and were not decompressed.

CPK elevated to 44,000 on post-op day 1 status post bilateral fasciotomies. The patient was managed on a bicarbonate drip until the CPK level began to drop and the rhabdomyolysis was medically managed. Post-op day 2, the CPK levels began to drop $(31,000)$, and she was kept on IV fluids with monitoring of CPK levels and renal function, and physical therapy was started. The patient was stable to undergo delayed primary closure of the fasciotomies on POD 3. CPK levels were monitored until they continued to decrease below 10,000 over the next several days.

The patient was then discharged from the hospital with a normal follow-up, and without any complications due to the compartment syndrome, the ensuing surgery or renal failure secondary to rhabdomyolysis.

\section{DISCUSSION}

The diagnosis of ACS is generally made clinically in the awake patient. In an obtunded patient the measurement of compartment pressures are helpful in establishing the diagnosis. This patient's presenting symptoms and physical examination findings of unusual pain coupled with limited/painful active and passive motion, paresthesias, and tense compartments, as well as lab findings consistent with rhabdomyolysis established the diagnosis [5]. The remaining "P's" of pallor, paresis, and pulselessness, (and some sources noting poikilothermia) are in fact late findings, and permanent damage may have already occurred [2]. Patients begin to experience pain when the intra-compartmental pressure reaches $20-30 \mathrm{mmHg}$ of diastolic blood pressure [6]. This makes 
pain an excellent indication and diagnostic clue to consider compartment syndrome in a differential diagnosis. However, the interpretation of compartment measurements continues to be a controversial topic, with some experts utilizing the difference between diastolic blood pressure and the compartment pressure to guide decision-making, as opposed to absolute compartment pressures alone[7]

Due to the limb-threatening nature of this phenomenon, early diagnosis and treatment is imperative. Six to eight hours is the current recommendation for decompression and restoration of muscle perfusion. Beyond this window the amount of salvageable muscle declines, and multiple subsequent debridements may be necessary. A recently published case report described the presentation of bilateral compartment syndrome in bilateral upper extremities 48 hours after the initial insult, where the patient experienced myonecrossis with resulting myoglobinuria that required intensive care monitoring and resuscitation, but did not progress to acute tubular necrosis and had no long-term deficits at six-month follow-up[8].

Soft tissue injury that results in a compartment syndrome with no other obvious signs/symptoms (such as trauma, broken bones) can cause a delay in treatment along with the development of complications that may result in permanent loss of function in a limb, or even limb loss [2]. Recent studies have shown that without a fracture, patients with compartment syndrome were delayed on average by over 12 hours to surgical fasciotomy (the definitive surgical therapy for compartment syndrome) [9].

In summary, acute compartment syndrome is a true medical and orthopedic surgical emergency that is generally diagnosed clinically, and requires immediate surgical intervention. Delay in diagnosis or treatment can cause permanent loss of function of or even the loss of the affected limb. Delays beyond the 6-8 hour window increase this risk greatly even if fasciotomy is performed. In this case, early diagnosis and surgical treatment with bilateral fasciotomies for decompression of both anterior and posterior compartments of the upper extremities was performed emergently. The use of the staple and vessel loop technique allowed for early delayed primary closure. This young woman's upper extremities recovered fully without any complications due to rapid diagnosis and surgical intervention. A high index of suspicion, clinical vigilance, and a willingness to act are imperative to avoid a missed or late compartment syndrome that can result in permanent damage to an extremity.

\section{REFERENCES}

1. J. G. Seiler III and S. P. Olvey, "Compartment syndromes of the hand and forearm," Journal of the American Society for Surgery of the Hand, vol. 3, no. 4, pp. 184-198, 2003.

2. Smith K, Wolford RW. Acute Idiopathic Compartment Syndrome of the Forearm in an Adolescent. Western Journal of Emergency Medicine. 2015;16(1):158-160. doi:10.5811/westjem.2014.9.23019.

3. Chopra R, Hayton M, Dunbar PJA. Exercise Induced Chronic Compartment Syndrome of the First Dorsal Interosseous Compartment of the Hand: A Case Report. Hand (New York, NY). 2009;4(4):415-417. doi:10.1007/s11552-009-9203-x.

4. Phillips JH, Mackinnon SE, Murray JF, McMurtry RY, et al. Exercise-induced chronic compartment syndrome of the first dorsal interosseous muscle of the hand: a case report. J Hand Surg. 1986;11A:124-27. And - Styf J, Forssblad P, Lundborg G. Chronic compartment syndrome in the first dorsal interosseous muscle. J Hand Surg. 1987;12A:757-62.

5. UfukSayar,TanılÖzer,andillker Mataracı, "ForearmCompartmentSyndromeCaused byReperfusionInjury,"Case Reports in Vascular Medicine, vol. 2014, Article ID 931410, 3 pages, 2014. doi:10.1155/2014/931410 
Exercise-Induced Acute Compartment Syndrome in Bilateral Upper Extremities: A Clinical Case

6. Tiwari A, Haq AI, Myint F, et al. Acute compartment syndromes. Br J Surg. 2002;89:397- 412.

7. McQueen M. M., Court-Brown C. M. Compartment monitoring in tibial fractures: the pressure threshold for decompression. Journal of Bone and Joint Surgery. 1996;78(1):99-104. [ubMed]

8. Traub BC, Lane MK, Traub JA. Exercise-Induced Acute Bilateral Upper-Arm Compartment Syndrome. Case Reports in Emergency Medicine. 2017;2017:9454782.doi:10.1155/2017/9454782.

9. Hope MJ, McQueen MM. Acute compartment syndrome in the absence of fracture. J Orthop Trauma. 2004;18(4):220-22.

Citation: Raymond E. Kennedy, MD, Mark K. Lane, MD. "Exercise-Induced Acute Compartment Syndrome in Bilateral Upper Extremities: A Clinical Case". American Research Journal of Orthopedics and Traumatology. 2018; 3(1): 1-4.

Copyright (c) 2018 Raymond E. Kennedy, MD, Mark K. Lane, MD. This is an open access article distributed under the Creative Commons Attribution License, which permits unrestricted use, distribution, and reproduction in any medium, provided the original work is properly cited. 